\title{
Solvable non-Hermitian discrete square well with closed-form physical inner product
}

\author{
Miloslav Znojil \\ Nuclear Physics Institute ASCR, \\ 25068 Řež, Czech Republic \\ e-mail: znojil@ujf.cas.cz
}

\begin{abstract}
A new Hermitizable quantum model is proposed in which the bound-state energies are real and given as roots of an elementary trigonometric expression while the wave function components are expressed as superpositions of two Chebyshev polynomials. As an $N$-site lattice version of square well with complex Robin-type two-parametric boundary conditions the model is unitary with respect to the Hilbert space metric $\Theta$ which becomes equal to the most common Dirac's metric $\Theta^{(\text {Dirac })}=I$ in the conventional textbook Hermitian-Hamiltonian limit. This metric is constructed in closed form at all $N=2,3, \ldots$.

\section{KEYWORDS}

exactly solvable quantum models;

discrete lattice;

non-Hermitian boundary conditions;

physical inner product;
\end{abstract}




\section{Introduction and summary}

Recent studies of quantum bound states in pseudo-Hermitian representations (cf., e.g., [1] for a review) revealed the existence of several paradoxes which are hidden in the conventional textbook perception of the theory. One of them is that we almost always select some "friendly" Hilbert space $\mathcal{H}^{(F)}$ of admissible states (typically, $\mathcal{H}^{(F)}=L^{2}(\mathbb{R})$ for a point particle) in advance. This implies that our choice of observables $\Lambda$ (including, in particular, Hamiltonians $H$ ) remains involuntarily restricted just to the class of the operators which are self-adjoint in this particular space.

Such a model-building strategy has been criticized and rejected by Bender et al who pointed out that it is far from universal (cf. review [2]). Via a family of Hamiltonians $H$ living in $\mathcal{H}^{(F)}=L^{2}(\mathbb{R})$ (with complex $g(x)=(i x)^{\delta}$ and real $\delta \in(0,2)$ in $H=p^{2}+g_{\delta}(x) x^{2} \neq H^{\dagger}$ ) these authors demonstrated (and persuaded the other quantum physicists) that for this type of models the conventional Hilbert space $L^{2}(\mathbb{R})$ is clearly unphysical, false and ill-chosen.

The resolution of such an $F$-space paradox has been found in nuclear physics [3, 4]. One comes to the conclusion that whenever $H \neq H^{\dagger}$ has a real spectrum, the "first", trivial inner

product of space $\mathcal{H}^{(F)}$ (which is called, by many authors, the "Dirac's" inner product) must be declared unphysical. What should follow is the introduction of an amended, "sophisticated" inner-product-metric operator $\Theta^{(S)}$, i.e., a change of the Hilbert space, $\mathcal{H}^{(F)} \rightarrow \mathcal{H}^{(S)}$. Thus, one may keep making calculations in $\mathcal{H}^{(F)}$ and upgrade merely the physical expectation values of observables $\Lambda$ in practice, $\langle\psi|\Lambda| \psi\rangle \rightarrow\langle\psi|\Theta \Lambda| \psi\rangle$.

The main role of the "second" metric $\Theta^{(S)} \neq I$ which defines the inner product in the physical Hilbert space lies in making the Hamiltonian self-adjoint in $\mathcal{H}^{(S)}$. The choice of the Hamiltonian and of the physical Hilbert space should proceed in parallel. The variability of both of the operators $H$ and $\Theta$ is only restricted by the constraint of Hermiticity imposed upon $H$ in $\mathcal{H}^{(S)}$, i.e., in the language of space $\mathcal{H}^{(F)}$, by the condition which has already been known to Dieudonné [5],

$$
H^{\dagger} \Theta^{(S)}=\Theta^{(S)} H
$$

Eq. (1) becomes a guide to a reversal of the conventional model-building strategy. Newly one starts from a given $H$ (which is non-Hermitian in unphysical $\mathcal{H}^{(F)}$ ) and inserts this operator and its adjoint in Eq. (11). Only then one solves the latter equation for unknown $\Theta^{(S)}$ and defines the correct physical Hilbert space $\mathcal{H}^{(S)}$.

Incidentally, the later recipe leads immediately to another, $S$-space paradox which has also been mentioned in Ref. [4]. Its essence is that a given Hamiltonian may be assigned many different metrics $\Theta=\Theta(H)$ via Eq. (1). Correctly, the authors of Ref. [4] pointed 
out that such an apparent paradox is in fact purely formal since a different metric would simply lead to different physics. With each new phenomenological candidate $\Lambda=\Lambda_{j}$ for an observable one has to require its Hermiticity in $\mathcal{H}^{(S)}$,

$$
\Lambda_{j}^{\dagger} \Theta^{(S)}=\Theta^{(S)} \Lambda_{j}, \quad j=1,2, \ldots, K
$$

As long as the metric operator $\Theta^{(S)}$ must remain the same during the process, one may expect the emergence of an unambiguous physical Hilbert space $\mathcal{H}^{(S)}$ at the end.

In our present paper we shall be guided by another, third paradox which may be noticed to lie in a deep conflict between the required gain in simplicity of the Hamiltonian $H \neq H^{\dagger}$ (highly appreciated, say, in the nuclear-physics applications of nontrivial metrics [4]) and the parallel loss of simplicity of the metrics $\Theta^{(S)} \neq I$. Indeed, although the latter loss is just the price to be paid for a broadening of the class of admissible observables (cf. [1]), the explicit construction of the metric usually appears almost prohibitively difficult in practice. In this sense it is only necessary to appreciate that the latter constructions, exact or approximate (cf., e.g., Refs. [6] for a few samples) still have been achieved for several, often fairly complicated systems in Hilbert spaces of infinite dimensions (cf., e.g., the remarkable and exceptional tractability of the "wrong-sign-oscillator" in [7]).

Still, the present-time scarcity of the available metrics in closed form is certainly one of the weakest points of the theory. For this reason we decided to search for as elementary an illustration of the theory as possible, and we found one. First of all, in a way inspired by the existing extensive literature on linear algebra (with applicability which is now very well accessible via the commercially available sophisticated software like MATHEMATICA or MAPLE) we succeeded because we restricted our choice of models to the ones represented by the matrices of arbitrary finite dimension $N$.

Naturally, we felt inspired also by a lot of work which was already done, in this direction, in physics (cf., e.g., its small sample in [8], with further references cited therein). In parallel, we were also guided by the existence of a few "exceptionally friendly" systems in mathematical physics [9] using linear algebra and matrices of arbitrary (sometimes even doubly infinite [10]) dimension.

Our results will be ordered as follows. First, the selection of the Hamiltonian $H$ will be discussed in section 2. For our model we will find the closed-form solution of Schrödinger equation in section 3 . The existence and localization of the subdomains $\mathcal{D}$ of physical parameters will be discussed, for which the spectra of bound state energies remain real and, hence, potentially observable. In section 4 we will then concentrate on the key technical 
problem of making the system unitary, i.e., on the constructive search for the missing metrics $\Theta=\Theta(H)$ in the form of an explicit $N$-parametric solution of Eq. (1).

The climax of the story will come with the observation that we shall be able to make the resulting physical Hilbert space $\mathcal{H}^{(S)}$ unique. This goal will be achieved via a less common though very natural requirement of the smoothness of the parallel limiting transition of operators $H$ and $\Theta(H)$ to their respective pre-determined special cases. More explicitly, in a way preferred by many authors (cf., e.g., [11]) we shall demand and guarantee that whenever the Hamiltonian becomes Hermitian, the related Hermitizing metric will acquire, in parallel, the conventional unit-operator Dirac's form of $\Theta^{(\text {Dirac })}=I$.

\section{Preliminaries}

\subsection{Differential square wells and their discrete descendants}

Ordinary and self-adjoint differential Schrödinger equation

$$
-\frac{d^{2}}{d x^{2}} \psi_{n}(x)=E_{n} \psi_{n}(x), \quad n=0,1, \ldots
$$

with the most common square-well boundary conditions

$$
\psi_{n}( \pm 1)=0
$$

offers one of the most transparent implementations of quantum theory [12]. Inter alia it may help in testing constructive methods and/or in sampling salient mathematical features of the formalism. In the former, testing context we may, for example, replace Eq. (3) by the sequence of its discrete, difference-equation descendants living on an equidistant and left-right symmetric lattice of $N=2 M-1$ grid points,

$$
\begin{array}{r}
-\psi_{n}\left(x_{k-1}\right)+2 \psi_{n}\left(x_{k}\right)-\psi_{n}\left(x_{k+1}\right)=E_{n}^{(M)} \psi_{n}\left(x_{k}\right), \\
k=-M+1,-M+2, \ldots, M-1 .
\end{array}
$$

This Schrödinger equation may be complemented, say, by the most common square well boundary conditions

$$
\psi_{n}\left(x_{ \pm M}\right)=0
$$

The exact solvability of all of these quantum bound state problems enables us to compare the $M=\infty$ spectrum of the ordinary differential Hamiltonian in (3) + (44) (leading to the ground-state energy $E_{0}=(\pi / 2)^{2} \approx 2.467$ and the first excited-state energy $E_{1}=\pi^{2} \approx 9.870$ 
etc) with the sequences of eigenvalues $E_{n}^{(M)}$ from Eqs. (15) $+(\underline{6})$ or rather with their tilded, properly rescaled values $\tilde{E}_{n}^{(M)}$ obtained after making the continuous and discrete systems compatible by fixing the discrete boundary points $x_{ \pm M}= \pm 1$.

A reasonably rapid $M \rightarrow \infty$ convergence to the continuous limit is revealed. With

$\tilde{E}_{0}^{(100)} \approx 2.492$ (and $\tilde{E}_{1}^{(100)} \approx 9.968$ etc [13]) the error-bar difference drops below one-percent at $M \approx 100$. Thus, in methodical setting the choice between differential Eq. (3) + (4) and its discrete analogue (5) $+(6)$ becomes just a matter of convenience. At the same time, the study of systems with finite $M$ will open multiple connections with the broad class of the so called constant-hopping chain models of condensed mater physics [8, 14, 15]. It is worth adding that the latter, unexpected correspondence would be lost if we had chosen any numerically more efficient version of the discretization.

\subsection{Making the differential square well models non-Hermitian}

The differential-difference and continuous-discrete parallels prove particularly relevant in the applicability context in which several interesting results were reported recently. For example, a few manifestly non-Hermitian upgrades of the square well were proposed and studied in Refs. [16, 17, 18]. In Ref. [19], attention has been redirected to the specific model which only lost its Hermiticity (though, incidentally, not the reality of the bound-state spectrum) due to an ad hoc complexification of boundary conditions,

$$
\psi( \pm 1)=\frac{\mathrm{i}}{\alpha} \frac{d}{d x} \psi( \pm 1), \quad \alpha \in \mathbb{R}
$$

The exact solvability of the model significantly contributed to the clarification of multiple open questions [20]. The analysis illustrated, in a broader methodical context, what happens when the Hermiticity of a Hamiltonian is replaced by a weaker though still phenomenologically tenable assumption of reality (i.e., in principle, observability) of the bound-state energy levels.

Before proceeding to a core of our present message, viz., to a deeper analysis of transitions from the differential-operator Eq. (3) to its difference-operator descendant (5) let us add that in Refs. [20], boundary conditions (7) were generalized to read

$$
\psi( \pm 1)=\frac{\mathrm{i}}{\alpha \mp \mathrm{i} \beta} \frac{d}{d x} \psi( \pm 1), \quad \alpha, \beta \in \mathbb{R} .
$$

From the point of view of physics, the resulting generalized non-Hermitian square-well boundstate problem proved much more interesting since at $\beta=0$ the spectrum was always real. Thus, only the choice of $\beta>0$ opened the phenomenologically most appealing possibility of 
having phase transitions, i.e., the spontaneous mergers and subsequent complexifications of colliding energy pairs. From the point of view of mathematics, unfortunately, the analysis of the general $\beta \neq 0$ case remained more or less purely numerical. This fact also contributed to motivation of our present analysis of the consequences of reduction of continuous Eq. (3) to discrete Eq. (15).

\section{Discrete non-Hermitian square well}

\subsection{Discretized boundary conditions (8)}

First of all, let us open the question of replacement of the Robin boundary conditions (8) by their discrete equivalent. For this purpose let us recall the first line of Eq. (5),

$$
-\psi_{n}\left(x_{-M}\right)+2 \psi_{n}\left(x_{-M+1}\right)-\psi_{n}\left(x_{-M+2}\right)=E_{n}^{(M)} \psi_{n}\left(x_{-M+1}\right)
$$

and the last line of Eq. (15),

$$
-\psi_{n}\left(x_{M-2}\right)+2 \psi_{n}\left(x_{M-1}\right)-\psi_{n}\left(x_{M}\right)=E_{n}^{(M)} \psi_{n}\left(x_{M-1}\right)
$$

and let us replace the complex Robin's differential boundary conditions (묘) by their respective first-difference analogues of the respective forms

$$
\begin{aligned}
\psi_{n}\left(x_{-M}\right) & =\frac{\mathrm{i}}{\alpha+\mathrm{i} \beta}\left(\frac{\psi_{n}\left(x_{-M+1}\right)-\psi_{n}\left(x_{-M}\right)}{h}\right), \\
\psi_{n}\left(x_{M}\right) & =\frac{\mathrm{i}}{\alpha-\mathrm{i} \beta}\left(\frac{\psi_{n}\left(x_{M}\right)-\psi_{n}\left(x_{M-1}\right)}{h}\right) .
\end{aligned}
$$

Here, a sufficiently small real constant $h>0$ stands for the difference between the neighboring points of the lattice. The insertion mediates the elimination of external $\psi_{n}\left(x_{-M}\right)$ and $\psi_{n}\left(x_{M}\right)$ and yields

$$
\begin{aligned}
& \left(2-E_{n}^{(M)}-\frac{1}{1-\beta h-i \alpha h}\right) \psi_{n}\left(x_{-M+1}\right)-\psi_{n}\left(x_{-M+2}\right)=0, \\
& -\psi_{n}\left(x_{M-2}\right)+\left(2-E_{n}^{(M)}-\frac{1}{1-\beta h+i \alpha h}\right) \psi_{n}\left(x_{M-1}\right)=0
\end{aligned}
$$

i.e., the two final forms of the upgraded, Robin-boundary-reflecting modification of the respective first and last line of our present toy-model Schrödinger Eq. (5).

It is worth adding here that besides the present discretization-approximation derivation and treatment of Eq. (5) (wherre, incidentally, the value of $z$ is never purely imaginary), an alternative physical background and meaning may be also assigned to such a model directly, 
say, via the current model-building strategies in condensed matter physics. For example, in Refs. [14, 15] (cf. also the related papers which are cited therein) the authors treat the similar tridiagonal-matrix models as an $N$-site tight-binding chain. In such a perspective they decided to work, for simplicity, just with the two purely imaginary $z$ treated as mutually conjugate single-site interactions. Even with such a constraint one reveals a wealth of interesting spectral phenomena. For example, the real (i.e., stable-system) spectrum may be fragile (cf. [15] and also 21] in this respect), while a remarkable "robust" exception occurs strictly in the end-point-interaction special case as studied in the earlier chain-model paper [14.

\subsection{Three domains of reality of spectra}

The replacement (3) $\rightarrow$ (5) of differential Schrödinger equation by its difference-equation analogue may be perceived as an approximation only in the limit of very small values of the lattice distance $h=x_{k+1}-x_{k}$ (or, if you wish, at the sufficiently large values of the number of grid points $N=2 M-1$ ). The same comment also applies to the parallel replacement of boundary conditions, e.g., (8) $\rightarrow$ (9), (10). This indicates that in the discrete models one could expect the occurrence of a richer spectral structure, at the larger values of the two variable parameters $\xi=\alpha h$ and $\zeta=\beta h$ at least.

The most straightforward verification of such a hypothesis may be performed numerically. Once we abbreviate $1 /(1-\beta h-i \alpha h)=z$ and $2-E_{n}^{(M)}=2 y$ we may rewrite our toy-model Schrödinger Eq. (15) + (11) + (12) in the compact matrix form

$$
\left[\begin{array}{ccccc}
2 y-z & -1 & 0 & \cdots & 0 \\
-1 & 2 y & -1 & \ddots & \vdots \\
0 & -1 & \ddots & \ddots & 0 \\
\vdots & \ddots & \ddots & 2 y & -1 \\
0 & \ldots & 0 & -1 & 2 y-z^{*}
\end{array}\right]\left[\begin{array}{c}
\phi_{1} \\
\phi_{2} \\
\vdots \\
\phi_{N-1} \\
\phi_{N}
\end{array}\right]=0 .
$$

Superscript ${ }^{*}$ denotes complex conjugation. In order to relax the artificial constraint $N=$ $2 M-1$ and to admit any $N$ by $N$ matrix Hamiltonian with $N=2,3, \ldots$ we also re-numbered the grid and the related wave-function values of $\psi_{n}\left(x_{-M+k}\right)=\phi_{k}$.

Even the first, preliminary numerical tests reveal that the qualitative features of the spectra will vary just smoothly with the matrix dimension. In particular, Fig. [1 shows that at $\beta=0$ the unbroken reality of the $N=\infty$ spectrum at all $\alpha$ [19] is strictly paralleled 


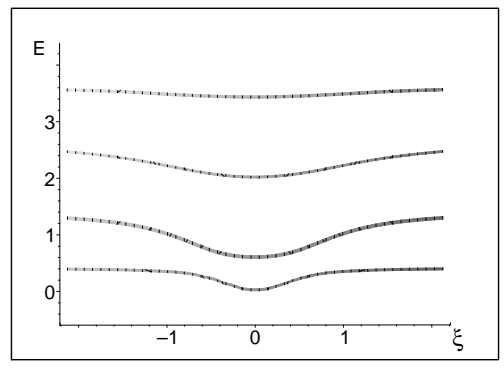

Figure 1: The $\xi$-dependence of the energies $E_{n}$ for the $\zeta=0$ Hamiltonian at $N=4$.

even by the first nontrivial discrete model using $N=4$. This example also shows that a perceivable effect of the deformation of spectrum of the conventional Hermitian $\xi \rightarrow \infty$ square well "of the second kind" [13] (in which $z=0$ ) is only obtained at comparatively small values of $\alpha$ alias $\xi$.

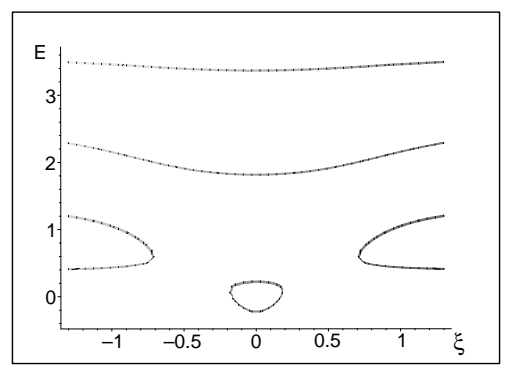

Figure 2: The supercritical $\xi$-dependent complexification of the first two lowest eigenvalues $E_{n}$ of the $\zeta=0.3$ Hamiltonian at $N=4$.

The well known $N=\infty$ observation of the complexification of the differential-operator spectra beyond certain critical values of parameters [22] is paralleled here by the behavior of the discretized models equally closely. In particular, beyond certain $\beta_{\text {critical }}>0$, all of the energies only remain real either at the truly very small values of $\alpha$ alias $\xi$ (e.g., at $|\xi| \lesssim 0.17$ in Fig. 2 where $N=4$ ) or in the two asymptotic, anomalous dynamical regimes of the large $|\xi|$ (e.g., roughly, with $\xi \gtrsim 0.7$ and $\xi \lesssim-0.7$ in Fig. 2).

The detailed analysis of the small $-\xi$ spectrum becomes particularly easy in the limit of $\xi=0$. Its study reveals that with the restricted growth of $\beta<1 / h$ alias $\zeta<1$ the allowed interval of small $\xi$ shrinks but still does not vanish. Surprisingly enough, the doublet of the two lowest real energies moves down to minus infinity in the limit $\zeta \rightarrow 1^{-}$. At $N=4$ this remarkable feature of the spectrum is documented in Fig. 3 which also displays a serendipitous symmetry of the spectrum with respect to the replacement of $\zeta \rightarrow 2-\zeta$. Naturally, these observations will also find their parallels after translation into the physical 


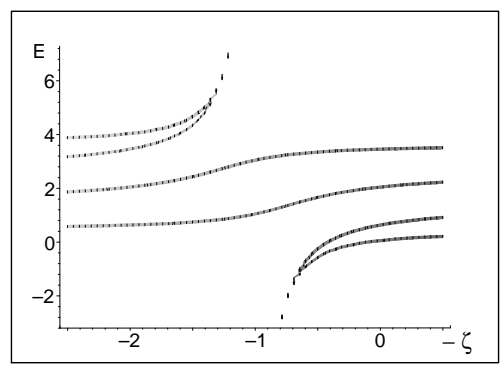

Figure 3: The $(-\zeta)$-dependence of the $N=4$ energies at $\xi=0$.

on-site-potential language of the phenomenological chain models of Refs. [14, 15].

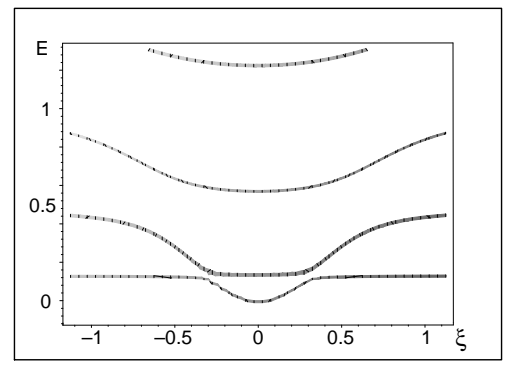

Figure 4: The subcritical $\xi$-dependence of the low-lying energies $E_{n}$ for the $\zeta=0.05$ Hamiltonian at $N=8$.

With the growth of dimension beyond $N=4$ just inessential, quantitative variations occur. Before the breakdown of the unconditional reality of the spectrum (i.e., at $\zeta<$ $\left.\zeta_{\text {critical }}(N)\right)$ the discrete models exhibit very similar $\xi$-dependence. During the growth of $|\xi|$ in the subcritical regime the two lowest energy levels get closer to each other (see Fig. 4 where we choose $N=8$ ). Subsequently (i.e., after the breakdown, at $\left.\zeta>\zeta_{\text {critical }}(N)\right)$, these two eigenvalues merge and become complex inside two $N$-dependent intervals of unphysical $\xi$ (cf. Figs. 2 or 5 where the complex levels are left invisible).

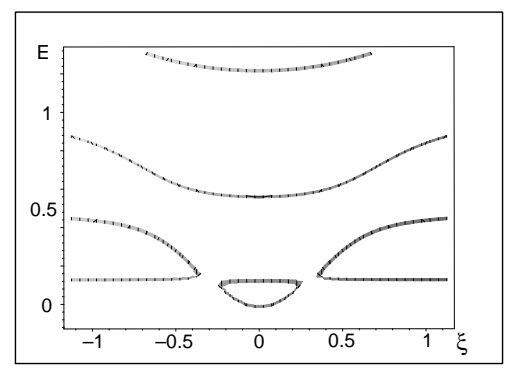

Figure 5: The supercritical $\xi$-dependence of the real low-lying energies $E_{n}$ for the $\zeta=0.07$ Hamiltonian at $N=8$. 
According to our numerical experiments with emphasis on the regime with small $\alpha$ alias $\xi$, the critical value of $\beta$ alias $\zeta=\zeta_{\text {critical }}(N)$ decreases with the dimension. As an illustration of this observation we may compare, e.g., $\zeta_{\text {critical }}(6)=0.09903$ (which we evaluated, by brute force, at $N=6$ ) with Figs. 4 and 5 which provide, at $N=8$, a rough bracketing information about $\zeta_{\text {critical }}(8) \in(0.05,0.07)$.

In the anomalous regime of the real spectrum at large $|\xi|$, one can also observe alternative complexifications during a decrease of $|\xi|$. In contrast to the previous case, these complexifications involve the higher excited pairs of levels at the higher values of $\zeta$. For illustration let us return to the $N=8$ model for which the small $-\xi$ complexification as shown in Fig. 5 took place at $\zeta=\zeta_{\text {critical }}(8)<0.07$ and concerned the lowest levels $E_{0}$ and $E_{1}$. We may add that in contrast to the decrease of the small $-\xi$ levels $E_{0}$ and $E_{1}$ with the growth of $\zeta$ as sampled in Fig. 3, the asymptotic loss of the reality of the energies during the decrease of the originally large $|\xi|$ moves upwards. It even involves higher energy excitations with the growth of $\zeta$. Thus, in the above $N=8$ example we noticed that near $\zeta=0.5$ the anomalous, decreasing $-\xi$ complexification is already being transferred from the first excited pair of $E_{1}$ and $E_{2}$ to the second excited pair of $E_{2}$ and $E_{3}$.

\subsection{The exact solvability of the model}

In Eq. (13) let us temporarily omit the first and last line. The resulting incomplete set

$$
\left[\begin{array}{cccccc}
-1 & 2 y & -1 & 0 & \ldots & 0 \\
0 & -1 & 2 y & -1 & \ddots & \vdots \\
\vdots & \ddots & \ddots & \ddots & \ddots & 0 \\
0 & \ldots & 0 & -1 & 2 y & -1
\end{array}\right]\left[\begin{array}{c}
\phi_{1} \\
\phi_{2} \\
\phi_{3} \\
\vdots \\
\phi_{N}
\end{array}\right]=0
$$

of the linear algebraic equations is formally solvable, in closed form, in terms of Chebyshev polynomials of the first and second kind [23],

$$
\phi_{n}=A T_{n-1}(y)+B U_{n-1}(y), \quad n=1,2, \ldots, N
$$

The two free (complex) parameters $A$ and $B$ may be restricted via a suitable normalization convention, say, $\phi_{1}=A+B=1$ (naturally, we cannot have a nontrivial solution such that $\left.\phi_{1}=0\right)$. Thus, we may eliminate $B=1-A$, get formula $\phi_{2}=(2-A) y$ and recall the 
temporarily omitted first line of Eq. (13) yielding the missing value of the second parameter $A=z / y$ in Eq. (15), provided only that $y \neq 0$.

The original Schrödinger Eq. (13) with $y=0$ must be considered separately. Taken as recurrences it gives

$$
\phi_{2 m-1}=(-1)^{m+1}, \quad \phi_{2 m}=(-1)^{m} z, \quad m=1,2, \ldots, \quad y=0 .
$$

Incidentally, once these formulae are perceived as the mere regularized $y \rightarrow 0$ limit of Eq. (15), we may conclude that the wave functions are known in closed form. Now, with the exceptional $y=0$ the last line of Eq. (13) imposes an additional constraint upon $z$. At even $N=2 K$ it reads $z^{*} z=1$ while at odd $N=2 K+1$ it has the form $z^{*}+z=0$.

At odd $N=2 K+1$ the discussion of the latter dichotomy is simpler because the solutions of the constraint $z^{*}+z=0=1-\beta h$ may be omitted as too singular (recall also Fig. 3 in this respect). At even $N=2 K$ the situation is less complicated. The assumption of vanishing $y=0$ means that our two free parameters must satisfy relation $z^{*} z-1=0=$ $(1-\beta h)^{2}+\alpha^{2} h^{2}-1$ which determines a circle in the $\alpha-\beta$ plane.

We may summarize that at "anomalous" $y=0$ the closed-form solution of our Schrödinger equation is completed. It is worth adding that at even $N=2 K$ and for very small $h$ and not too large $\alpha$ s the circle of parameters degenerates, locally, to a line, $-2 \beta=\mathcal{O}(h)$. This implies the constancy of $\beta=0$ in the limit $h \rightarrow 0$. Thus, we still stay in a standard dynamical regime. For example, let us recall that in Fig. 1 where $K=2$, the second excited level $E_{2}=2-2 y_{2}$ has strictly vanishing $y_{2}=0$ at vanishing $\xi=\alpha h=0$.

We may now return to the determination of the other bound-state energy eigenvalues $y=y_{k} \neq 0$ for which Schrödinger equation is also reduced to the single algebraic relation represented by the last line of Eq. (13). After all insertions this "non-anomalous" relation acquires the form

$$
\frac{z}{y} T_{N-2}(y)+\left(1-\frac{z}{y}\right) U_{N-2}(y)=\left(2 y-z^{*}\right)\left[\frac{z}{y} T_{N-1}(y)+\left(1-\frac{z}{y}\right) U_{N-1}(y)\right]
$$

admitting simplification

$$
-z U_{N-3}(y)+U_{N-2}(y)=\left(2 y-z^{*}\right)\left[-z U_{N-2}(y)+U_{N-1}(y)\right]
$$

and acquiring the final representation

$$
z^{*} z U_{N-2}(y)-\left(z+z^{*}\right) U_{N-1}(y)+U_{N}(y)=0
$$

This is secular equation which determines the energy-representing variables $y_{n}=1-E_{n} / 2$ as roots of a real polynomial. This means that these roots are either real or forming complex 
conjugate pairs. One can easily check, by direct insertion, that this equation also leads to the correct answer for the above-discussed (and temporarily eliminated) "anomalous" solutions for which one had $y_{n}=0$. Secondly, in the regime of very small parameters we may set $z \approx 1$ in Eq. (19) and get

$$
U_{N-2}\left(y_{s}\right)-2 y_{s} U_{N-1}\left(y_{s}\right)+U_{N}\left(y_{s}\right)=2\left(1-y_{s}\right) U_{N-1}\left(y_{s}\right)=0 .
$$

Thus, the set of roots of this special limiting case is composed of the separate real item $y_{s}=1$ and of the $(N-1)$-plet of the well known and non-degenerate $N-1$ real roots of $U_{N-1}(z)$ which lie inside interval $(-1,1)$. This enables us to deduce, rigorously, that a sufficiently small perturbation due to the presence of non-vanishing parameters $\xi \approx 0$ and $\zeta \approx 0$ will still keep even the slightly deformed $N$-plet of the roots real since their possible complexifications may merely proceed via their pairwise confluences.

In this manner we practically completed the proof of the following exact-solvability result.

Proposition 1. For our discrete non-Hermitian square well Schrödinger Eq. (13) the wave functions are given, at any dimension $N=2,3, \ldots$ and complex $z$, by formula (15) or by its $y \rightarrow 0$ limit (16). The reparametrization $y=\cos \gamma$ of the bound-state energy variables leads to the elementary trigonometric secular equation

$$
z^{*} z \sin (N-1) \gamma-\left(z+z^{*}\right) \sin N \gamma+\sin (N+1) \gamma=0, \quad 0 \leq \gamma<\pi
$$

Moreover, for the sufficiently small parameters $\xi$ and $\zeta$ in $z=1 /(1-\zeta-i \xi)$, the $N-$ plet of the energy roots $y_{n}=\cos \gamma_{n}$ will be real and non-degenerate.

Proof. Eq. (21) resulted from Eq. (19) after multiplication by $\sin \gamma$. As long as we may assume that $0 \leq \gamma \leq \pi$, just the endpoints of this interval with $y= \pm 1$ have to be reconsidered, therefore. Knowing that $U_{n}(1)=n+1$ while $U_{n}(-1)=(-1)^{n}(n+1)$, the respective special cases of the correct secular Eq. (19) have the form

$$
\left(z^{*} \mp 1\right)(z \mp 1) N=z^{*} z-1 \text {. }
$$

This just restricts the choice of $z$ at a given $N$. More explicitly, with $\zeta=\beta h$ and $\xi=\alpha h$ we have $z=1 /(1-\zeta-i \xi)$ so that $z z^{*}=1 /\left[(1-\zeta)^{2}+\xi^{2}\right]$ and $z+z^{*}=2(1-\zeta) /\left[(1-\zeta)^{2}+\xi^{2}\right]$.

In the first step we have to re-analyze our correct secular equation at $y=1$,

$$
\frac{N-1}{(1-\zeta)^{2}+\xi^{2}}-\frac{2 N(1-\zeta)}{(1-\zeta)^{2}+\xi^{2}}+N+1=0 .
$$

This leads to the elimination of

$$
\xi^{2}=-\zeta^{2}+\frac{2 \zeta}{N+1}
$$


so that our $y=1$ solution [i.e., the obviously existing $\gamma=0$ solution of Eq. (21)] will be acceptable whenever

$$
0 \leq \zeta \leq \frac{2}{N+1}
$$

This is compatible with our parameter-smallness assumptions.

In the second step we set $y=-1$ and obtain the constraint

$$
\frac{N-1}{(1-\zeta)^{2}+\xi^{2}}+\frac{2 N(1-\zeta)}{(1-\zeta)^{2}+\xi^{2}}+N+1=0 .
$$

For positive $\xi=\rho \sin \delta$ and $\zeta=\rho \cos \delta$ with $0 \leq \delta \leq \pi / 2$ this enables us to eliminate

$$
\sin \delta=\frac{4 N+(N+1) \rho^{2}}{(4 N+2) \rho}
$$

and treat $\rho$ as a free parameter which keeps the angle $\delta$ real if and only if

$$
2-\frac{2}{N+1} \leq \rho \leq 2 \text {. }
$$

We see that either $\xi$ or $\zeta$ cannot be small in such a case. Thus, by inequality $\gamma<\pi$ the artificially added $(N+1)$-st root $\gamma=\pi$ of Eq. (21) was crossed out again as redundant, in the small-parametric dynamical regime at least.

\section{The metrics}

At any matrix dimension $N<\infty$ and for the physical parameters guaranteeing the reality of the spectrum of $H$ the determination of any acceptable (i.e., Hermitian and positive-definite) matrix solution $\Theta$ of Dieudonné's Eq. (11) is rarely feasible and always ambiguous. Even in the feasible cases (cf., e.g., [24]) the knowledge of an output of virtually any preliminary symbolic-manipulation calculation hardly helps and often provides just long and illegible formulae. This makes the constructions of the metrics rather challenging even at the very small dimensions $N$.

\subsection{All the metrics at $N=3$}

For the sake of simplicity let us just consider the simplest dynamical $\zeta=0$ scenario and Hamiltonian

$$
H=\left[\begin{array}{ccc}
-(1-i \xi)^{-1} & -1 & 0 \\
-1 & 0 & -1 \\
0 & -1 & -(1+i \xi)^{-1}
\end{array}\right]
$$


All of the possible related metrics may be sought in the form of a six-parametric complex ansatz

$$
\Theta=\left[\begin{array}{ccc}
r & u+i u_{2} & z_{1}+i z_{2} \\
u-i u_{2} & s & u+i u_{2} \\
z_{1}-i z_{2} & u-i u_{2} & r
\end{array}\right]
$$

Its insertion converts Dieudonné's Eq. (11) into a set of nine (not necessarily independent) relations with a complete three-parametric solution

$$
\begin{gathered}
u_{2}=-\frac{r \xi}{1+\xi^{2}}, \quad z_{2}=-\frac{\left(u \xi^{2}+r+u\right) \xi}{\left(1+\xi^{2}\right)^{2}} \\
z_{1}=\frac{s-r+u+2 s \xi^{2}-3 r \xi^{2}-\xi^{4} r+\xi^{4} s+u \xi^{2}}{\left(1+\xi^{2}\right)^{2}} .
\end{gathered}
$$

Obviously, even though we choose just the first nontrivial dimension $N=3$, an exhaustive discussion of all of the resulting optional kinematical scenarios looks prohibitively complicated. In fact, it need not be necessarily so: a few constructive samples of the analysis of what happens to the complete sets of operators of observables under nontrivial metrics may be found, e.g., in [25].

Whenever $N \geq 3$, a practically necessary guide to simplifications of our present constructions of metrics has been found in the idea that whenever the Hamiltonian $H$ becomes self-adjoint in $\mathcal{H}^{(F)}$ at some special parameters (i.e., in the limit of $\xi \rightarrow 0$ in our case), all of the metrics $\Theta$ which remain, in the same limit, different from the "natural" Dirac's trivial $\Theta^{(F)}=I$ might be discarded as anomalous and pathological. On this basis we set $s=r=1$ and got the special, simplified one-parametric family of the $N=3$ metrics possessing a nicer and more compact form

$$
\Theta(u)=\left[\begin{array}{ccc}
1 & u-\frac{i \xi}{1+\xi^{2}} & \frac{u-\xi^{2}+u \xi^{2}}{1+2 \xi^{2}+\xi^{4}}-\frac{i\left(u \xi^{2}+1+u\right) \xi}{\left(1+\xi^{2}\right)^{2}} \\
u+\frac{i \xi}{1+\xi^{2}} & 1 & u-\frac{i \xi}{1+\xi^{2}} \\
\frac{u-\xi^{2}+u \xi^{2}}{1+2 \xi^{2}+\xi^{4}}+\frac{i\left(u \xi^{2}+1+u\right) \xi}{\left(1+\xi^{2}\right)^{2}} & u+\frac{i \xi}{1+\xi^{2}} & 1
\end{array}\right] .
$$

This matrix is amenable to numerical testing. One reveals that for any $u \neq 0$, the $\xi \rightarrow 0$ limit of such a matrix will differ from the isotropic Dirac's $\Theta^{(F)}=I$. For this reason we finally set $u=0$. The resulting matrix $\Theta(0)$ proved tractable as a metric because it remains positive definite in the whole physical (i.e., real-energies-guaranteeing) range of parameter $\xi \in(-\infty, \infty)$. 


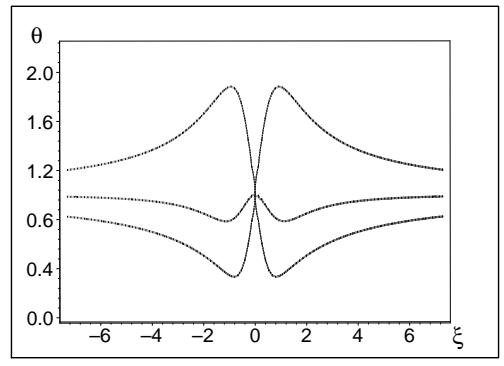

Figure 6: The $\xi$-dependence of eigenvalues of the $N=3$ metric (23) with $s=r=1$ and $u=0$.

Fig. [6] displays the $\xi$-dependence of the triplet of the metric's eigenvalues. One should add that up to a constant factor, the smoothness of transition $\Theta(0) \rightarrow \Theta^{(F)}=I$ happens to be guaranteed not only in the "natural" Hermitian-Hamiltonian limit of small $\xi \rightarrow 0$ but also, beyond the scope of Fig. 6, in the other two Hermitian-Hamiltonian limits $\xi \rightarrow \pm \infty$. In this sense metric $\Theta(0)$ may be considered optimal.

\subsection{Special metric at $N=4$}

Along the same lines as in preceding subsection one may insert the adjusted general $N=4$ ansatz for $\Theta$ and insert it again in Eq. (1). The computer-assisted symbolic-manipulation solution of this set of $N^{2}$ linear algebraic equations is routine. It yields the formulae (too long for a display here) which contain now as many as four independent free real parameters.

It proved rather fortunate that the extrapolated, parameter-free simplified ansatz

$$
\Theta=\left[\begin{array}{cccc}
1 & u_{1}+i u_{2} & z_{1}+i z_{2} & r_{1}+i r_{2} \\
u_{1}-i u_{2} & 1 & u_{1}+i u_{2} & z_{1}+i z_{2} \\
z_{1}-i z_{2} & u_{1}-i u_{2} & 1 & u_{1}+i u_{2} \\
r_{1}-i r_{2} & z_{1}-i z_{2} & u_{1}-i u_{2} & 1
\end{array}\right]
$$

with units on its main diagonal appeared optimal again. Not too surprisingly, the limiting $\xi \rightarrow 0$ case would remain anisotropic for $u_{1} \neq 0$. Nevertheless, the choice of $u_{1}=0$ reestablished the isotropy. Moreover, the related simplification left the result of the symbolicmanipulation solution of Eq. (1) printable, yielding

$$
\begin{gathered}
u_{2}=-\frac{\xi}{1+\xi^{2}}, \quad z_{1}=-\frac{\xi^{2}}{\left(1+\xi^{2}\right)^{2}}, \quad z_{2}=-\frac{\xi}{\left(1+\xi^{2}\right)^{2}} \\
r_{1}=-2 \frac{\xi^{2}}{\left(1+\xi^{2}\right)^{3}}, \quad r_{2}=-\frac{\left(-\xi^{2}+1\right) \xi}{\left(1+\xi^{2}\right)^{3}} .
\end{gathered}
$$


Last but not least, it was important to find that also all the four eigenvalues of the resulting unique metric remained positive and finite in the whole physical range of the dynamical parameter $\xi \in(-\infty, \infty)$ and that they all proved equal in all of the three alternative Hermitian limits of $\xi \rightarrow 0$ and $\xi \rightarrow \pm \infty$ (cf. Fig. (7).

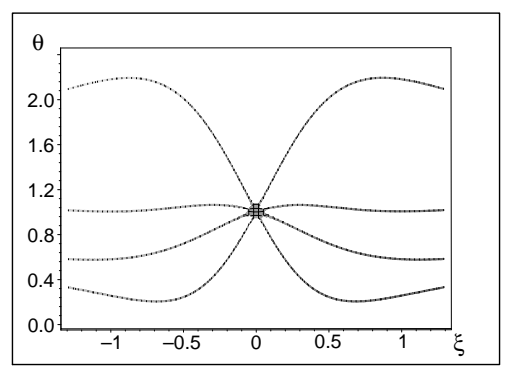

Figure 7: The eigenvalues of metric (25) with $u_{1}=0$.

\subsection{A reparametrization of Hamiltonians}

One of the remarkable features of matrix elements (26) is that they vanish in all of the three Hermiticity limits, i.e., in the extremes of both small and large parameters $\xi$. Naturally, one pays for the universality by the complicated algebraic form of these formulae.

There exist several reasons why one should not pay too much attention to the two rather artificial large $-\xi$ physical domains. Naturally, they do not have any direct $N \rightarrow \infty$ limiting connection with the continuous Schrödinger Eq. (3) with the complex Robin boundary conditions (7) or (8). In this sense we shall assume, from now on, that both of the parameters $\xi$ and $\zeta$ in the Hamiltonian $H(\xi, \zeta)$ of Eq. (13) remain small and that they may be replaced by another real and small pair, say, $(\omega, \varrho)$, with the maximal attention paid to the resulting simplification of the matrices in question.

As long as the realization of such a trick involves just the two extreme elements in our Hamiltonians, it is sufficient to illustrate it at $N=3$. Thus, we decided to require that

$$
H=\left[\begin{array}{ccc}
-z(\zeta, \xi) & -1 & 0 \\
-1 & 0 & -1 \\
0 & -1 & -z(\zeta,-\xi)
\end{array}\right]=\left[\begin{array}{ccc}
-1-\varrho-i \omega & -1 & 0 \\
-1 & 0 & -1 \\
0 & -1 & -1-\varrho+i \omega
\end{array}\right]
$$

i.e.,

$$
z(\zeta, \xi)=\frac{1}{1-\zeta-i \xi}=1-\varrho+i \omega
$$


An easy algebra leads to the formulae

$$
\begin{gathered}
\omega=\omega(\zeta, \xi)=\frac{\xi}{(1-\zeta)^{2}+\xi^{2}}, \\
\varrho=\varrho(\zeta, \xi)=\frac{\zeta-\zeta^{2}-\xi^{2}}{(1-\zeta)^{2}+\xi^{2}} .
\end{gathered}
$$

Although such a reparametrization $(\xi, \zeta) \rightarrow(\omega, \varrho)$ of the whole real plane is rather complicated globally, we simply have the trivial mapping $\omega \sim \xi$ and $\varrho \sim \zeta$ in one of the most interesting physical domains near the origin. Secondly, in the condensed-matter context of Refs. [14, 15] our reparametrization (28) of the interaction may be also perceived as establishing a more direct connection between our mathematical results and the physics of the stable $\mathcal{P} \mathcal{T}$-symmetric condensed-matter $N$-site lattices containing a pair of weak impurities localized at the two ends.

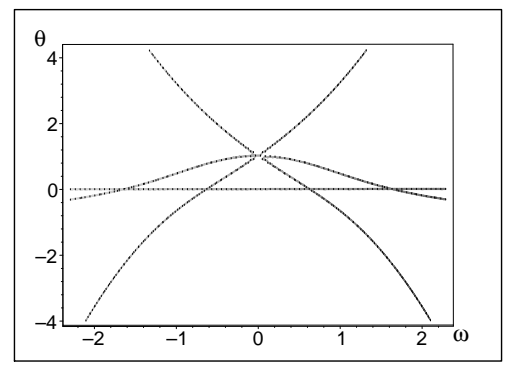

Figure 8: Eigenvalues of metric (31) at $\varrho=0$. The loss of positivity with the growth of $|\omega|$.

Besides the natural physical and perturbation-theory motivated interest in the parametric domain near the origin, the decisive advantage of our reparametrization lies in the enormous simplification of the related exact matrices of the metrics. For example, at $\varrho=0$ the righthand-side Hamiltonian matrix of Eq. (27) is now being assigned the exact $u=0$ Hermitizing metric

$$
\Theta^{(3)}=\left[\begin{array}{ccc}
1 & -i \omega & -\omega^{2}-i \omega \\
i \omega & 1 & -i \omega \\
-\omega^{2}+i \omega & i \omega & 1
\end{array}\right] .
$$

A routine check confirms that at the small values of $\omega$ this matrix is positive definite as it should be. According to Fig. 8 this matrix ceases to be positive definite at certain larger $\omega_{\text {critical }}$ but this just reflects the fact that the energy spectrum of the Hamiltonian itself ceases to be real at these points which lie comparatively far from the origin. One should add that this does not contradict Figs. 1 and/or 6, either, because due to Eq. (30) the line of $\varrho=0$ is represented by a comparatively large circle in the $\zeta-\xi$ plane. 
The even more persuasive advantages of our reparametrization emerge during a transition to the larger dimensions $N$. Thus, the next, $N=4$ and $\varrho=0$ Hamiltonian

$$
H=\left[\begin{array}{cccc}
-1-i \omega & -1 & 0 & 0 \\
-1 & 0 & -1 & 0 \\
0 & -1 & 0 & -1 \\
0 & 0 & -1 & -1+i \omega
\end{array}\right]
$$

is being assigned the $u=0$ metric

$$
\Theta^{(4)}=\left[\begin{array}{cccc}
1 & -i \omega & -\omega^{2}-i \omega & -2 \omega^{2}+i\left(\omega^{3}-\omega\right) \\
i \omega & 1 & -i \omega & -\omega^{2}-i \omega \\
-\omega^{2}+i \omega & i \omega & 1 & -i \omega \\
-2 \omega^{2}-i\left(\omega^{3}-\omega\right) & -\omega^{2}+i \omega & i \omega & 1-t h
\end{array}\right]
$$

with the spectrum which is fully analogous to the one of Fig. 8 above.

\subsection{Metrics at all matrix dimensions $N$}

On the background of preceding preparatory considerations there emerges a pattern which may be made explicit and formulated as our second main result.

Proposition 2. For the special and reparametrized one-parametric $\varrho=0$ subset

$$
H^{(N)}(\omega)=\left[\begin{array}{ccccc}
-1-i \omega & -1 & 0 & \ldots & 0 \\
-1 & 0 & -1 & \ddots & \vdots \\
0 & \ddots & \ddots & \ddots & 0 \\
\vdots & \ddots & -1 & 0 & -1 \\
0 & \ldots & 0 & -1 & -1+i \omega
\end{array}\right]
$$

of our $N$ by $N$ matrix Hamiltonians of Eq. (13) with $N \geq 2$ the closed form of Hermitizing metric $\Theta^{(N)}(\omega)$ with the Dirac-limit property $\Theta^{(N)}(0)=I$ is given by formulae $\Theta_{n n}^{(N)}=1$, $n=1,2, \ldots, N$ and

$$
\Theta_{n n+k}^{(N)}=\left(\Theta_{n+k, n}^{(N)}\right)^{*}=-i \omega(1-i \omega)^{k-1}, \quad n=1,2, \ldots, N-k, \quad k=1,2, \ldots, N-1
$$


Proof. The choice of $\Theta_{n n}^{(N)}=1$ with $n=1,2, \ldots, N$ fixes some of the available free parameters so that we just have to prove the validity of formulae (34). This result is trivial at $N=2$ and it has been shown to hold at $N=3$ and $N=4$ in the preceding text (cf. Eqs. (31) and (32), respectively). It remains for us to show that the metric $\Theta^{(N)}(\omega)$ and matrix $H$ of Eq. (33) are mutually compatible with respect to the Dieudonné's constraint (1) at any $N$.

For our present purposes the latter constraint may be perceived as the $N$ by $N$ matrix set of linear algebraic equations $\mathcal{M}_{i j}^{(N)}=0$. Thus, starting from a generic ansatz

$$
\begin{gathered}
\Theta_{n n}^{(N)}=\left(\Theta_{n n}^{(N)}\right)^{*}=p_{1}^{(1)}+i p_{2}^{(1)}, \quad \Theta_{n n+1}^{(N)}=\left(\Theta_{n+1, n}^{(N)}\right)^{*}=p_{1}^{(2)}+i p_{2}^{(2)}, \ldots \\
\ldots \Theta_{n, N}^{(N)}=\left(\Theta_{N, n}^{(N)}\right)^{*}=p_{1}^{(N)}+i p_{2}^{(N)}
\end{gathered}
$$

we immediately see that the knowledge of the "old" matrix elements $p_{1}^{(N-j)}+i p_{2}^{(N-j)}$ with $j=1,2, \ldots, N-1$ leaves just the following four nontrivial complex relations in Eq. (11),

$$
\mathcal{M}_{i j}^{(N)}=0, \quad(i, j) \in\{(1, N-1),(2, N),(N-1,1),(N, 2)\} .
$$

They are, incidentally, linearly dependent. Thus, taking any one of these relations, we have to split it into its real and imaginary parts. This yields the pair of the real recurrence relations

$$
p_{1}^{(N)}=p_{1}^{(N-1)}+\omega p_{2}^{(N-1)}, \quad p_{2}^{(N)}=p_{2}^{(N-1)}-\omega p_{1}^{(N-1)} .
$$

A patient mathematical induction may then start from the known initial values of

$$
p_{1}^{(1)}=1, \quad p_{2}^{(1)}=0
$$

or, if you wish, from one of the above-mentioned explicit-calculation results with

$$
p_{1}^{(2)}=0, \quad p_{2}^{(2)}=-\omega, \quad p_{1}^{(3)}=-\omega^{2}, \quad p_{2}^{(3)}=-\omega
$$

etc. The elementary combinatorics finally proves the step $N-1 \rightarrow N$.

Remark 1. From the formal point of view, the restriction of our attention to the metrics with all of the free parameters fixed by the condition $\Theta^{(N)}(0)=I$ may still have many well founded alternatives. We would like to add that in the case of need of any more flexible family of metrics, the extreme simplicity of our Hamiltonians may be expected to admit still some closed-form alternative constructions in which $\Theta^{(N)}(0) \neq I$.

Their first sample is the following 
Proposition 3. The same $N$ by $N$ matrix Hamiltonians (33) as above may be assigned the closed-form Hermitizing metric $\Theta^{(N)}(\omega)=\Theta_{(0)}^{(N)}(\omega)+u \Theta_{(1)}^{(N)}(\omega)$ where $\Theta_{(0)}^{(N)}(\omega)$ denotes the $u=0$ metric of Proposition 2. Closed formulae define $\left(\Theta_{(1)}\right)_{n n}^{(N)}=0, n=1,2, \ldots, N$ and

$$
\left(\Theta_{(1)}\right)_{n n+k}^{(N)}=\left[\left(\Theta_{(1)}\right)_{n+k, n}^{(N)}\right]^{*}=(1-i \omega)^{k-1}, \quad n=1,2, \ldots, N-k, \quad k=1,2, \ldots, N-1 .
$$

At each $N$ the value of parameter $u$ must remain sufficiently small to keep the metric positive definite.

Proof. Naturally, once we use the amended guess of the form of matrix elements of metric, its direct insertion is easily shown to convert also the above-mentioned non-trivial components of the set of Dieudonnian recurrences (35) into identities.

In an alternative to the above proof, we could still use an entirely general ansatz and proceed by mathematical induction as above. Even in such a more traditional approach the verification of compatibility between metric and Hamiltonian may be facilitated by the use of computer-assisted symbolic manipulations. 


\section{References}

[1] A. Mostafazadeh, Int. J. Geom. Meth. Mod. Phys. 7 (2010) 1191.

[2] C. M. Bender, Rep. Prog. Phys. 70 (2007) 947.

[3] F. J. Dyson, Phys. Rev. 102 (1956) 1217.

[4] F. G. Scholtz, H. B. Geyer and F. J. W. Hahne, Ann. Phys. (NY) 213 (1992) 74.

[5] J. Dieudonne, Proc. Int. Symp. Lin. Spaces, Pergamon, Oxford, 1961, pp. 115-122.

[6] M. S. Swanson, J. Math. Phys. 45 (2004) 585;

A. Mostafazadeh, J. Phys. A: Math. Gen. 39 (2006) 10171;

A. Mostafazadeh, J. Math. Phys. 47 (2006) 072103;

A. Mostafazadeh, J. Phys. A: Math. Gen. 39 (2006) 13506;

F. G. Scholtz and H. B. Geyer, Phys. Lett. B 634 (2006) 84;

C. Figueira de Morisson Faria and A. Fring, Czech J. Phys. 56 (2006) 899;

C. Figueira de Morisson Faria and A. Fring, J. Phys. A: Math. Gen. 39 (2006) 9269;

P. Musumbu-Dibwe, H. G. Geyer, W. D. Heiss, J. Phys. A: Math. Gen. 40 (2007) F75;

C. Quesne, J. Phys. A: Math. Gen. 40 (2007) F745;

P. E. G. Assis and A. Fring, J. Phys. A: Math. Theor. 41 (2008) 244001;

C. Quesne, J. Phys. A: Math. Theor. 41 (2008) 244022;

P. E. G. Assis and A. Fring, J. Phys. A: Math. Theor. 42 (2009) 015203;

H. F. Jones, J. Phys. A: Math. Theor. 42 (2009) 135303;

S. Dey, A. Fring and B, Khantoul, J. Phys. A: Math. Theor. 46 (2013) 335304;

A. Ghatak and B. P. Mandal, Comm. Theor. Phys. 59 (2013) 533.

[7] V. Buslaev and V. Grechi, J. Phys. A: Math. Gen. 26 (1993) 5541.

[8] Ch. Korff and R. A. Weston, J. Phys. A: Math. Theor. 40 (2007) 8845;

M. Znojil, J. Phys. A: Math. Theor. 40 (2007) 4863;

O. A. Castro-Alvaredo and A. Fring, J. Phys. A: Math. Theor. 42 (2009) 465211; 
O. Bendix, R. Fleischmann, T. Kottos and B. Shapiro, Phys. Rev. Lett. 103 (2009) 030402;

L. Jin and Z. Song, Comm. Theor. Phys. 54 (2010) 73;

M. Znojil, Phys. Rev. A 82 (2010) 052113;

S. Longhi, Phys. Rev. A 88 (2013) 052102;

B.-G. Zhu, R. Lu and S. Chen, Phys. Rev. A 89 (2014) 062102.

[9] M. Znojil, J. Math. Phys. 50 (2009) 122105;

E. Ergun and M. Saglam, Rep. Math. Phys. 65 (2010) 367.

[10] M. Znojil, SIGMA 5 (2009) 085.

[11] H. Bíla, PhD thesis, Charles University (2008) (listing older references);

H. Bíla, Adiabatic time-dependent metrics in PT-symmetric quantum theories. arXiv:0902.0474 (unpublished).

[12] A. Messiah, Quantum Mechanics, North Holland, Amsterdam, 1961.

[13] M. Znojil, Phys. Lett. A 375 (2011) 2503.

[14] L. Jin and Z. Song, Phys. Rev. A 80 (2009) 052107.

[15] Y. N. Joglekar, D. Scott, M. Babbey and A. Saxena, Phys. Rev. A 82 (2010) 030103(R).

[16] H. Langer and Ch. Tretter, Czech. J. Phys. 54 (2004) 1113;

P. Siegl, Int. J. Theor. Phys. 50 (2011) 991.

[17] M. Znojil, Phys. Lett. A 285 (2001) 7;

M. Znojil and G. Lévai, Mod. Phys. Letters A 16 (2001) 2273;

M. Znojil, J. Phys. A: Math. Gen. 39 (2006) 441.

[18] A. Mostafazadeh and A. Batal, J. Phys. A: Math. Theor. 37 (2004) 11645.

[19] D. Krejcirik, H. Bila and M. Znojil, J. Phys. A 39 (2006) 10143.

[20] J. Zelezny, Int. J. Theor. Phys. 50 (2011) 1012;

D. Krejcirik, P. Siegl, and J. Zelezny, Compl. Anal. Oper. Theory 8 (2014) 255. 
[21] M. Znojil, J. Math. Phys. 45 (2004) 4418.

[22] G. Lévai and M. Znojil, Mod. Phys. Letters A 16 (2001) 1973;

D. Krejcirik, P. Siegl, M. Tater and J. Viola, submitted (arXiv:1402.1082).

[23] M. Abramowitz and I. A. Stegun, Handbook of Mathematical Functions, Dover, New York, 1970.

[24] D. Krejcirik, J. Phys. A: Math. Theor. 41 (2008) 244012.

[25] M. Znojil, J. Phys. Conf. Ser. 343 (2012) 012136;

M. Znojil, Ann. Phys. (NY) 327 (2012) 893. 